\title{
Ligand-Assisted Reconstruction of Colloidal Quantum Dots Decreases Trap State Density
}

Bin Sun ${ }^{1}$, Maral Vafaie ${ }^{1}$, Larissa Levina ${ }^{1}$, Mingyang $\mathrm{Wei}^{1}$, Yitong Dong ${ }^{1}$, Yajun $\mathrm{Gao}^{2}$, Hao Ting Kung $^{3}$, Margherita Biondi ${ }^{1}$, Andrew H. Proppe ${ }^{1,5}$, Bin Chen ${ }^{1}$, Min-Jae Choi ${ }^{1}$, Laxmi Kishore

Sagar ${ }^{1}$, Oleksandr Voznyy, ${ }^{1}$ Shana O. Kelley ${ }^{4,5}$, Frédéric Laquai ${ }^{2}$, Zhenghong Lu$^{3}$, Sjoerd Hoogland $^{1}$, F. Pelayo García de Arquer ${ }^{1}$, Edward H. Sargent ${ }^{1 *}$

${ }^{1}$ Department of Electrical and Computer Engineering, University of Toronto, 10 King's College Road, Toronto, Ontario, M5S 3G4, Canada

${ }^{2}$ King Abdullah University of Science and Technology (KAUST), KAUST Solar Center (KSC), Physical Sciences and Engineering Division (PSE), Material Science and Engineering Program (MSE), Thuwal 23955-6900, Kingdom of Saudi Arabia

${ }^{3}$ Department of Material Science and Engineering, University of Toronto, 184 College St, Toronto, Ontario, M5S 3E4, Canada

${ }^{4}$ Department of Pharmaceutical Science, Leslie Dan Faculty of Pharmacy, University of Toronto, Toronto, ON, M5S 3G4, Canada.

${ }^{5}$ Department of Chemistry, University of Toronto, Toronto, Ontario, Canada, M5S 3G4

${ }^{4}$ Department of Material Science and Engineering, University of Toronto, 184 College St, Toronto, Ontario, M5S 3E4, Canada

*E-mail: ted.sargent@utoronto.ca

KEYWORDS: Quantum dots, Surface defects, Trap states, Dimers, Photovoltaics 
Colloidal quantum dots (CQDs) are of interest in solar cell applications in view of their widely-tunable bandgap, controlled via nanocrystal size. Increasing the power conversion efficiency (PCE) of CQD solar cells has relied on improving the passivation of surfaces in the CQD solid, enhancing CQD coupling and charge transport, and advancing device architecture. The presence of hydroxyl groups on the nanoparticle surface, as well as dimers - fusion between CQDs - have been found to be the major source of trap states, detrimental to optoelectronic properties and device performance. Here we introduce a CQD reconstruction step that decreases surface hydroxyl groups and dimers simultaneously. We explored the dynamic interaction of charge carriers between bandedge states and trap states in CQDs using time-resolved spectroscopy, showing that trap-to-ground-state recombination occurs mainly from surface defects in coupled CQD solids passivated using simple metal halides. Using CQD reconstruction, we demonstrate a $60 \%$ reduction in trap density and a $25 \%$ improvement in charge diffusion length. These translate into a PCE of 12.5\% compared to $\mathbf{1 0 . 9 \%}$ for control untreated CQDs. 
Colloidal quantum dots (CQDs) have attracted interest in solar cell applications in view of their widely tunable bandgap and their solution processing. ${ }^{1-6}$ Device efficiencies have been increased via improved ligand exchanges, ${ }^{7,8}$ increased CQD homogeneity and coupling, ${ }^{9}$ and new device architectures. ${ }^{10,11}$ The best performance CQD solar cell has been built on a bulk heterojunction active layer-separating photoexcited electrons and holes into distinct phases, achieving a PCE of $13.3 \% .^{12}$

We posited that the quality of as-synthesized including CQDs polydispersity and surface defects continues to impact the density of trap states, which influences the open-circuit voltage ${ }^{13}$, 14 and also the charge-carrier recombination. ${ }^{15}$ Lead chloride treatment has been reported to reported to improve monodispersity of PbS CQD ensembles. ${ }^{16}$ However, the application of $\mathrm{PbCl}_{2}$ treated CQDs in optoelectronic devices has not been reported; this may be due to the reduced carrier transport caused by $\mathrm{PbCl}_{\mathrm{x}}$ Shell. ${ }^{17-19}$ Recently, epitaxial dimers - pairs of CQDs fused together with an epitaxially-registered crystal structure at their interface - have been found to be major sources of trap states in thiol-based-ligand-passivated PbS CQD solids. ${ }^{20}$

Additionally, Zherebetskyy et al. reported that hydroxyl (OH) groups stay on PbS (111) facets during synthesis when using $\mathrm{PbO}$ as precursor. ${ }^{21}$ Halide anions $(\mathrm{Cl}-, \mathrm{Br}-$, and $\mathrm{I}-)$ and molecular halides passivation have been reported as an effective route to reduce the density of dangling bonds and trap states by replacing organic long ligands, leading to improved PCE. ${ }^{4,7,17}$, ${ }^{22,}{ }^{23}$ Unfortunately, $\mathrm{OH}$ groups are not readily removed during the ligand exchange process, and they end up acting as surface trap states in CQD solids. ${ }^{24}$ It has been previously reported that employing $\mathrm{PbAc}_{2}$ and $\mathrm{PbX}_{2}\left(\mathrm{X}_{=} \mathrm{Cl}^{-}, \mathrm{Br}^{-} \text {, and } \mathrm{I}^{-}\right)^{25-28}$ instead of $\mathrm{PbO}$ during the preparation of the lead oleate precursor leads to the partially removal of $\mathrm{OH}$ groups during synthesis. ${ }^{25}$ However, the residual $\mathrm{OH}$ was enough to limit the PCE to under $11 \% .25,28$

Here we report a surface reconstruction process that decreases the number of $\mathrm{OH}$ surface groups and dimers using halides at the cooling stage of the synthesis. At the end of synthesis ligand digestive ripening ${ }^{29}$ and Ostwald ripening ${ }^{30}$ are in competition with one another by adding ligands. Ligand digestive ripening narrows CQD size distribution with the help of ligands that dissolve large particles completely - releasing monomers for the growth of the smaller sized ensembles. ${ }^{31}$ Ostwald ripening is the inverse process, wherein small particles are dissolved. ${ }^{32}$ By controlling these two processes we reconstruct the CQDs, achieving improved CQD monodispersity (size 
dispersity $2.7 \%$ ) and reduced density of $\mathrm{OH}$ groups on CQD surfaces. We reduce the density of trap states by $60 \%$, mainly due to fewer surface defects, confirmed by tracking trap to ground-state recombination using time-resolved spectroscopy. The decreased surface defects also led to a longer carrier lifetime and diffusion lengths, translating to CQD solar cells exhibiting higher PCEs of $12.5 \%$, compared to $10.9 \%$ for untreated CQDs. In general, our results not only revealed the major source of trap states in metal-halide-passivated CQD solids but also provided a new path to further improve the CQD quality via CQD reconstruction process.

a)

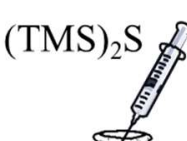

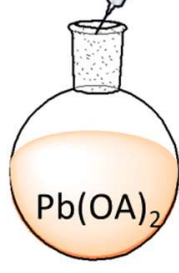

$110^{\circ} \mathrm{C}$
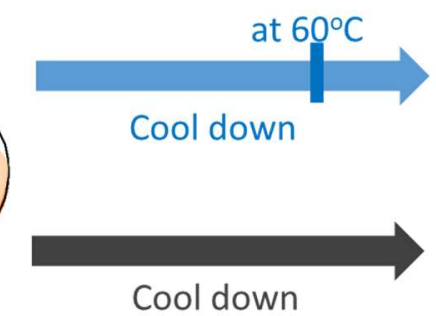

Cool down

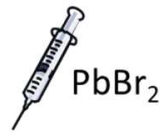

$\mathrm{PbBr}_{2}$
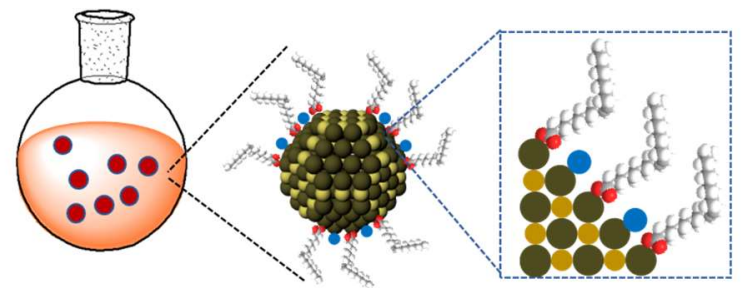

$\mathrm{Pb}$
$\mathrm{S}$
$\mathrm{Br}$
$\mathrm{C}$
$\mathrm{O}$
$\mathrm{H}$

b)

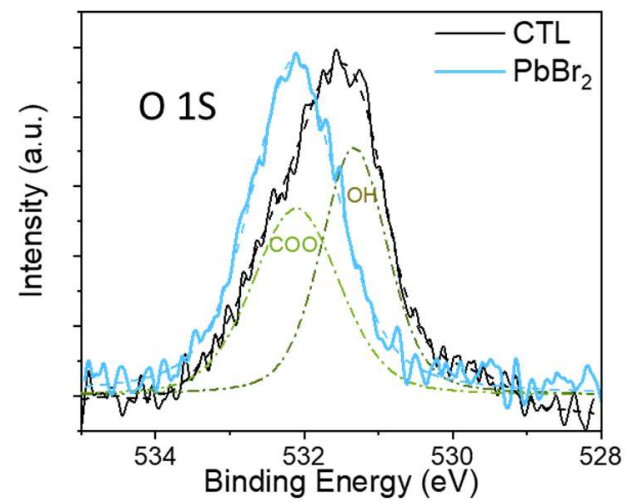

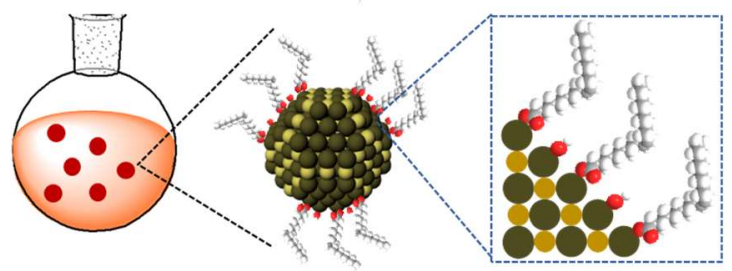

c)

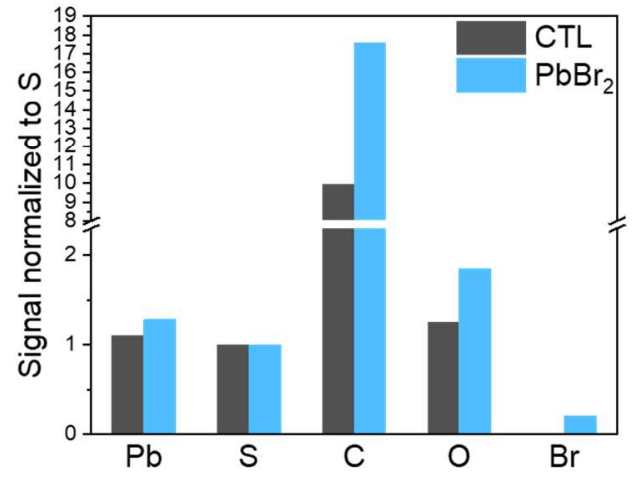

Figure 1. Improved surface passivation via $\mathrm{PbBr}_{2}$ reconstruction. a) $\mathrm{PbBr}_{2}$ in-synthesis reconstruction; b) $\mathrm{O}$ 1s signal from CQDs with and without $\mathrm{PbBr}_{2}$ reconstruction; c) elemental ratios of CQDs with and without $\mathrm{PbBr}_{2}$ reconstruction extracted from XPS.

\section{Results}


We synthesized the CQDs following a modified method from Hines et al. ${ }^{33}$ When the CQD solution was cooled to $60{ }^{\circ} \mathrm{C}$ near the end of the synthesis, $\mathrm{PbBr}_{2}$ in oleylamine ${ }^{34}$ was injected in the CQD solution (Figure 1a). The $\mathrm{Br}^{-}$anions function as an etching agent, ${ }^{35}$ enabling the ripening process to reconstruct CQDs and improve the size distribution.

In order to thoroughly understand how the $\mathrm{PbBr}_{2}$ reconstruction affects the CQDs surface properties, we performed XPS analyses on the synthesized CQDs. We observed a dramatically reduced amount of $\mathrm{OH}$ groups and the presence of $\mathrm{Br}^{-}$on the $\mathrm{CQD}$ surface following the reconstruction (Figure 1b). The CTL-PbS CQDs XPS spectrum contains two peaks at 532.1 and $531.3 \mathrm{eV}$, corresponding to the carboxyl (COO) and $\mathrm{OH}$ groups respectively, in agreement with the previously reported surface-OH groups..$^{21,25}$ In contrast, the $\mathrm{OH}$ signal is undetectable for the CQDs after $\mathrm{PbBr}_{2}$ reconstruction $\left(\mathrm{PbBr}_{2}-\mathrm{PbS}\right)$. After integrating the $\mathrm{O}$ 1s peak for CTL-PbS in Figure $1 \mathrm{~b}$, the molar ratio of $\mathrm{O}$ from $\mathrm{COO}$ and from $\mathrm{OH}$ is $\sim 1$. We then extracted the elemental ratios from XPS (Figure 1c), where the signals are normalized to the $\mathrm{S} 2 \mathrm{p}$ peak. The O:S ratio is 1.25 for CTL-PbS, (0.625 from $\mathrm{COO}$ and 0.625 from $\mathrm{OH})$. The $\mathrm{O}: \mathrm{S}$ ratio is 1.85 for $\mathrm{PbBr}_{2}-\mathrm{PbS}$, where $1.225(1.85-0.625)$ is approximately the additional $\mathrm{COO}$ after reconstruction $(\sim 100 \%$ increased O:S ratio by replacing $\mathrm{OH}$ with $\mathrm{COO}$ ). This suggests that most of the $\mathrm{OH}$ is replaced by COO, leading to $\sim 200 \%$ more oleic acid ligands on PbS surfaces and better passivation. The CTL$\mathrm{PbS}$ and $\mathrm{PbBr}_{2}-\mathrm{PbS}$ show a $\mathrm{Pb}: \mathrm{S}$ ratio of 1.1 and 1.28 , respectively. The $\mathrm{PbBr}_{2}-\mathrm{PbS}$ has a higher $\mathrm{Pb}: \mathrm{S}$ ratio due to the existence of $\mathrm{Br}^{-}\left(\mathrm{PbBr}_{3}^{-}\right)$passivation layers.

In order to study the CQD size distribution via the reconstruction, we performed scanning transmission electron microscopy (STEM) measurements for CTL-PbS (Figure 2a) and $\mathrm{PbBr}_{2-}$ PbS CQDs (Figure 2b). The STEM images show highly monodispersed CQDs with a mean diameter of $\sim 3.13 \pm 0.14 \mathrm{~nm}$ for the CTL-PbS and $\sim 3.14 \pm 0.12$ the $\mathrm{PbBr}_{2}-\mathrm{PbS}$ CQDs by analyzing $\sim 150$ of QDs (Figure 2c, dimers are not counted). However, a higher number of dimers (the concentration of dimers is $\sim 1$ per 100 QDs, from TEM results) can be observed in the STEM images of CTL-PbS (Figure 2a \& S1), compared to the $\mathrm{PbBr}_{2}-\mathrm{PbS}$ CQDs, where no dimers are observed in the STEM images. We attribute the reduced number of dimers to the digestive ripening mechanism, which causes larger nanoparticles to slowly dissolve in favor of smaller ones via etching and stabilization of the surfaces by $\mathrm{Br}^{-}$ligands. ${ }^{31}$ 
To study the effect of the reduced OH groups and the decreased dimers, we performed absorbance (Abs) and photoluminescence (PL) measurements (Figure 2d). The half-width at half maximum (HWHM) value of the first excitonic peak is $70 \mathrm{meV}$ and $60 \mathrm{meV}$ for CTL-PbS and $\mathrm{PbBr}_{2}-\mathrm{PbS}$ respectively (Figure S2). These values correspond to a decrease in size dispersity from $3.0 \%$ for CTL-PbS to $2.7 \%$ for $\mathrm{PbBr}_{2}-\mathrm{PbS}$ CQDs, ${ }^{36}$ which is close to the smallest value reported for $\mathrm{PbSCQDs} .{ }^{16} \mathrm{The}_{\mathrm{PbBr}}$ reconstruction also results in an increase of the peak-to-valley ratio from 2.9 to 4.0 and improves the PL spectrum full width at half maximum (FWHM) value (134 $\mathrm{meV}$ for $\mathrm{PbBr}_{2}-\mathrm{PbS}$ and $155 \mathrm{meV}$ for CTL-PbS, Figure S2). These improvements are attributed to the reduction of the number of dimers following the $\mathrm{PbBr}_{2}$ reconstruction, in agreement with the STEM results.

a)

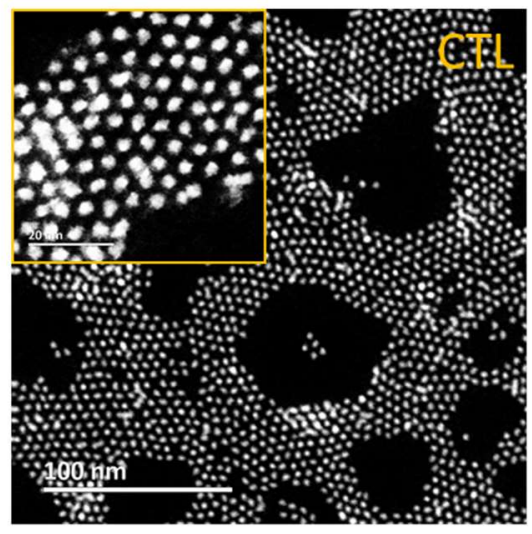

b)
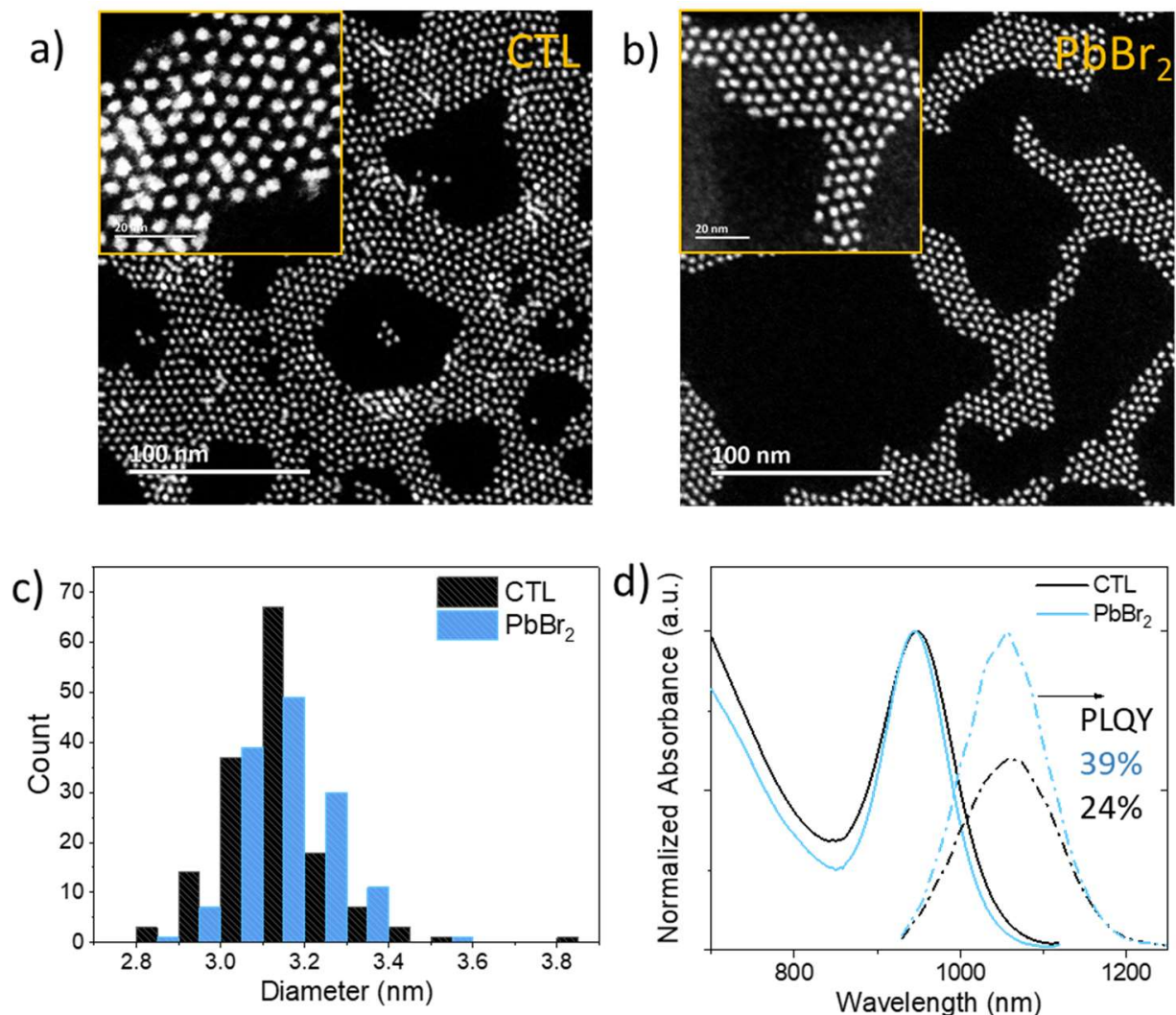

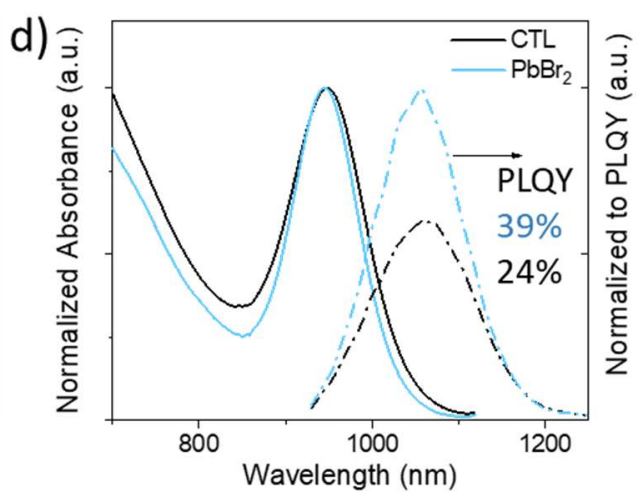

Figure 2. Reduced size distribution via $\mathbf{P b B r} 2$ reconstruction. STEM micrograph of a) CTL$\mathrm{PbS}$ ( $\sim 20$ dimers out of $\sim 2000$ QDs from the STEM) and b) $\mathrm{PbBr}_{2}-\mathrm{PbS}$ CQDs with a zoom-in view inset (inset scale bar, $20 \mathrm{~nm}$ ); c) STEM extracted particle size distribution histograms for the CTL$\mathrm{PbS}$ and $\mathrm{PbBr}_{2}-\mathrm{PbS}$ samples, d) absorption and photoluminescence of CTL-PbS and $\mathrm{PbBr}_{2}-\mathrm{PbS}$ CQDs solution. 
Because of the combination of improved surface passivation and monodispersity, the photoluminescence quantum yield (PLQY) of the CQDs improves from $24 \%$ to $39 \%$ for $\mathrm{PbBr}_{2-}$ $\mathrm{PbS}$ (Figure S3). This is an indicator of reduced trap density, ideal for photovoltaic applications.

Considering these promising results, we studied the optoelectronic properties of CQD solids fabricated with ligand exchanged CQDs. We used a previously reported one-step solutionphase ligand exchange to replace the long oleic acid ligands with halides. ${ }^{7}$ OA-CQDs were ligandexchanged from the nonpolar octane phase into the polar N,N-dimethylformamide (DMF) phase containing $\mathrm{PbBr}_{2}, \mathrm{PbI}_{2}$, and ammonium acetate $\left(\mathrm{NH}_{4} \mathrm{Ac}\right)$. We performed XPS measurements (Figure S4) on exchanged CQD films and found that the organic long ligands were replaced by lead halide (Br- and I-) ligands. ${ }^{7}$ A broad O 2p peak for CTL-PbS CQD solid (Figure S4a) following ligand exchange was also observed by XPS, indicating the residual $\mathrm{OH}$ groups from the assynthesized CTL-PbS CQDs; in contrast, the $\mathrm{PbBr}_{2}-\mathrm{PbS}$ CQD films show a sharp O $2 \mathrm{p}$ peak, corresponding to carboxyl (COO) from oleic acid and acetic acid ligands. We also carried out absorption measurements on exchanged CQDs (Figure $\mathrm{S} 4 \mathrm{c}$ ). The exchanged $\mathrm{PbBr}_{2}-\mathrm{PbS}$ CQDs show a better monodispersity in both DMF solution and films, indicating that the low size distribution is maintained after ligand exchange.

We employed field-effect transistor (FET) measurements to characterize the CQDs transport properties. ${ }^{8}$ We utilized a bottom-gate top-contact FET configuration, where $70 \mathrm{~nm}$ of Ti was deposited on the glass as the gate electrode, followed by $15 \mathrm{~nm}$ of $\mathrm{ZrO}_{2}$ for a gate dielectric providing a gate capacitance of $460 \mathrm{nF} \mathrm{cm}$. A thin $100 \mathrm{~nm}$ CQD film was deposited on the $\mathrm{ZrO}_{2}$ gate dielectric, and $100 \mathrm{~nm} \mathrm{Au}$ source and drain electrodes were deposited by thermal evaporation (Figure 3a). FET transfer characteristics reveal n-type transport for both CTL-PbS and $\mathrm{PbBr}_{2}-\mathrm{PbS}$ CQD films (Figure 3b). We calculated carrier mobility from the slope of the drain-source current $\left(I_{D S}\right)$ vs. gate voltage $\left(V_{G S}\right)$ according to the equation $I_{D S}=\mu C_{i} W L\left(V_{G S}-V_{T H}\right) V_{D S}(1)$, where $\mu$ is the carrier mobility in the linear regime; $I_{D S}$ is the drain-source current; $C_{i}$ is the gate dielectric layer capacitance per unit area; $L$ and $W$ are the channel length $(50 \mu \mathrm{m})$ and channel width $(2.5 \mathrm{~mm})$ respectively; and $V_{G S}$ and $V_{T H}$ are the gate voltage and threshold voltage, respectively. CTL-PbS and $\mathrm{PbBr}_{2}-\mathrm{PbS}$ films yielded similar electron mobility values of $0.028 \pm 0.002 \mathrm{~cm}^{2} \mathrm{~V}^{-1} \mathrm{~s}^{-1}$ and $0.029 \pm 0.003 \mathrm{~cm}^{2} \mathrm{~V}^{-1} \mathrm{~s}^{-1}$, respectively. 

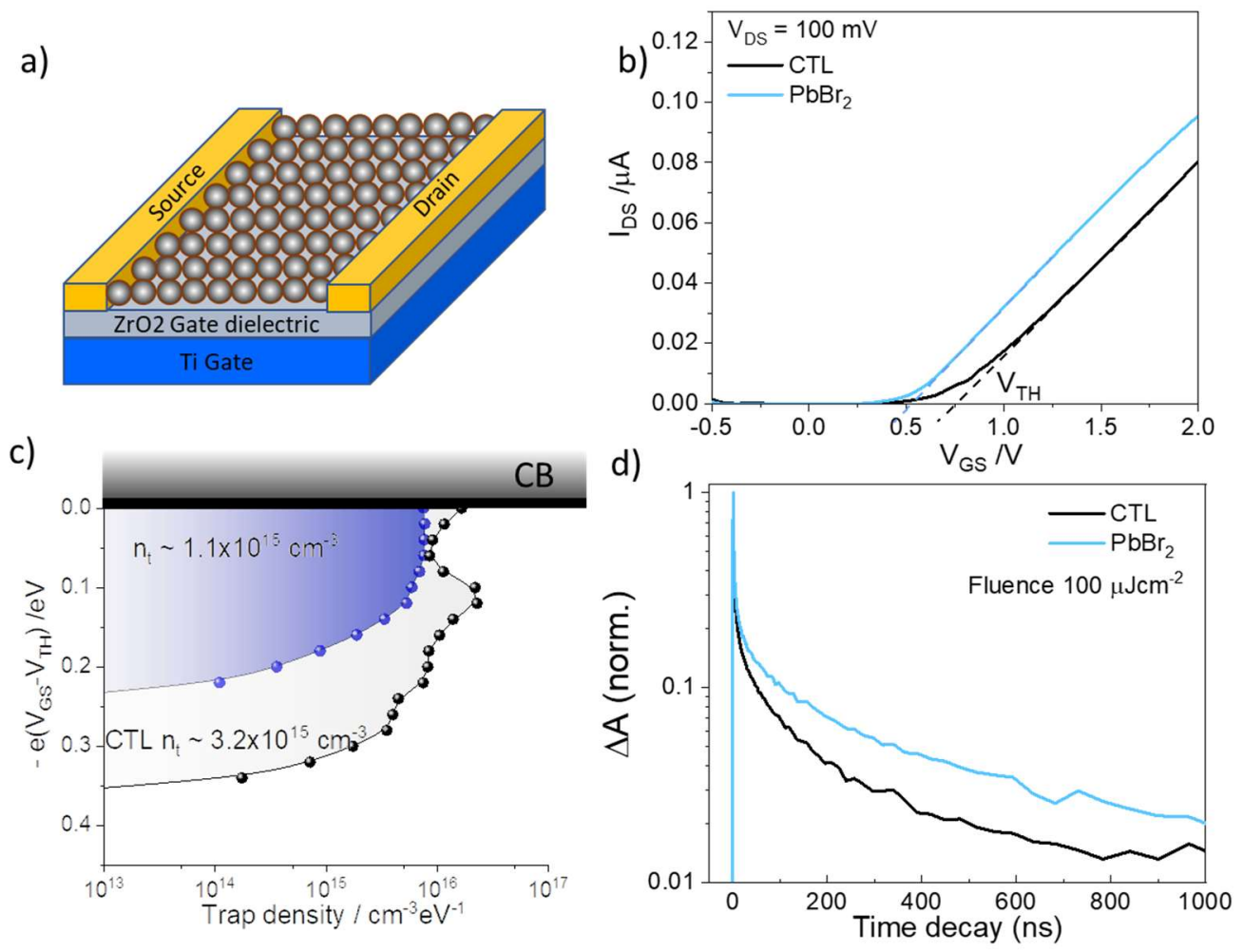

Figure 3. Improved transport properties of PbBr2-PbS CQD films. a) Bottom-gate top-contact field-effect transistor structure; b) transfer characteristics of exchanged PbS CQDs with/without $\mathrm{PbBr}_{2}$ reconstruction; c) density of trap states $\left(N_{\mathrm{T}}\right)$ of exchanged $\mathrm{PbBr}_{2}-\mathrm{PbS}$ (blue) and CTL-PbS (grey) films as calculated with Eq (4) identified close to the conduction bands. d) Kinetic traces (taken from the maximum of the band-edge exciton bleaching signal) of CTL-PbS and $\mathrm{PbBr}_{2}-\mathrm{PbS}$ CQD films after ligand exchange.

We then used transient absorption (TA) measurements to ascertain the carrier lifetime. ${ }^{37}$ TA experiments were performed on different samples with photoexcitation fluences ranging from 5 to $500 \mu \mathrm{J} \mathrm{cm}^{-2}$. Transients at the same fluence for the two different samples are shown in Figure 3d and Figure S5 (other traces can be found in Figure S6). We observe a rapid decay within the first $10 \mathrm{~ns}$ when using stronger pump fluences due to the increased Auger recombination. ${ }^{38}$ After $10 \mathrm{~ns}$, the signal decays at slower rates and becomes slower with 
increasing fluence, likely due to the trap-state filling. ${ }^{39}$ The lifetimes extracted from these measurements are consistent with previously reported lifetimes for PbS CQD solids. ${ }^{39,40}$ For all fluences, the lifetime of $\mathrm{PbBr}_{2}-\mathrm{PbS}$ film is longer than that of CTL-PbS, indicating longer carrier lifetimes in the $\mathrm{PbBr}_{2}-\mathrm{PbS}$ films.

We extracted the lifetime from the TA results (Figure S5) and calculated the diffusion length $L_{d}{ }^{39,41}$ by the following formula

$$
L_{d}=\sqrt{\mathrm{D} \tau}
$$

where $\mathrm{D}$ is the diffusion coefficient and $\tau$ is the carrier lifetime. $\mathrm{D}$ is related to carrier mobility via the Einstein relation: $\mathrm{D}=\frac{k T \mu}{q}$ (3), where $k$ is the Boltzmann constant, $T$ is temperature, $\mu$ is the carrier mobility, and $q$ is the charge of the carrier. The $\mathrm{PbBr}_{2}-\mathrm{PbS}$ films have a $L_{d}$ of $150 \pm 5 \mathrm{~nm}$, more than $25 \%$ longer than that of the CTL-PbS film $(120 \pm 4 \mathrm{~nm})$, when the lifetime is extracted from TA data pumped by $100 \pm 5 \mu \mathrm{J} \mathrm{cm}^{-2}$. $\mathrm{PbBr}_{2}-\mathrm{PbS}$ films always show longer $L_{d}$ compared to the one of the CTL-PbS films under different fluence power, where $\mathrm{PbBr}_{2}-\mathrm{PbS}$ films show longer carrier lifetime with different influence (Figure S7).

We also retrieved the density of in-gap trap states from measured transfer characteristics of FETs to confirm if the as-synthesized highly monodispersed CQDs lead to a decreased density of trap states in the exchanged CQD films, ${ }^{8,42}$ since the gate capacitance $\left(460 \mathrm{nF} \mathrm{cm}{ }^{-2}\right)$ is large enough to shift the semiconductor active layer Fermi energy by several hundred millivolts. Varying the gate bias over a range of few hundred millivolts allowed us to map the Fermi energy shift from the exponential increase of the drain current from $V_{\text {ON }}$ to $V_{\text {TH }}$ (Figure $S 8,3 c$ ) in the subthreshold regime below $V_{\mathrm{TH}}$, which corresponds to transport through trap states, to obtain a trap state distribution following the equation:

$$
N_{t}=\left[\left(\frac{S \cdot e}{k T \cdot \ln (10)}-1\right) \cdot \frac{C_{i}}{e}\right]^{2} \cdot \epsilon_{0} \epsilon_{r}^{-1}
$$

where $S$ is a sub-threshold swing (Figure S8), the inverse of the subthreshold slope of the $\log$ drain current versus the gate voltage below $V_{T H}$ that defines the boundary between the subthreshold and transport regime; $\epsilon_{0}$ is the vacuum permittivity; and $\epsilon_{r}$ is the electric constant of the film, estimated to be $10.9 .{ }^{43}$ After integrating the tail state distribution between the subthreshold and transport regime (Figure 3b ) for the $\mathrm{PbBr}_{2}-\mathrm{PbS}$ films, we obtained the 
density of tail states $\left(N_{\mathrm{T}}\right)$ (plotted in Figure 3c). The exchanged $\mathrm{PbBr}_{2}-\mathrm{PbS}$ CQD film exhibits a trap density $N_{\mathrm{T}}$ of $1.5 \pm 0.3 \times 10^{15} \mathrm{~cm}^{-3}$, lower than that of the CTL-PbS films, $5.0 \pm 0.4 \times 10^{15}$ $\mathrm{cm}^{-3}$, which agrees with the above results of the longer carrier lifetime and diffusion lengths.
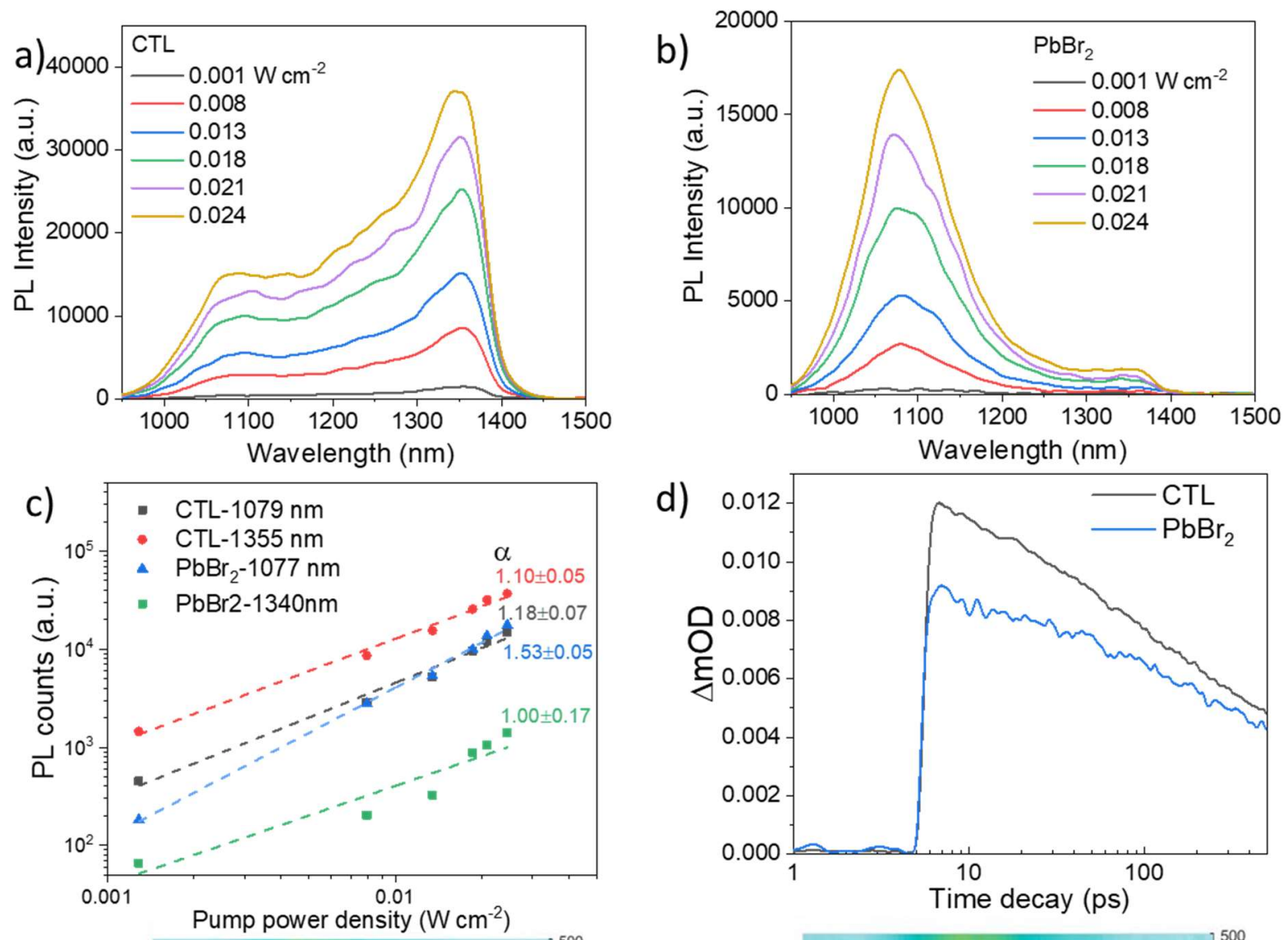

d)
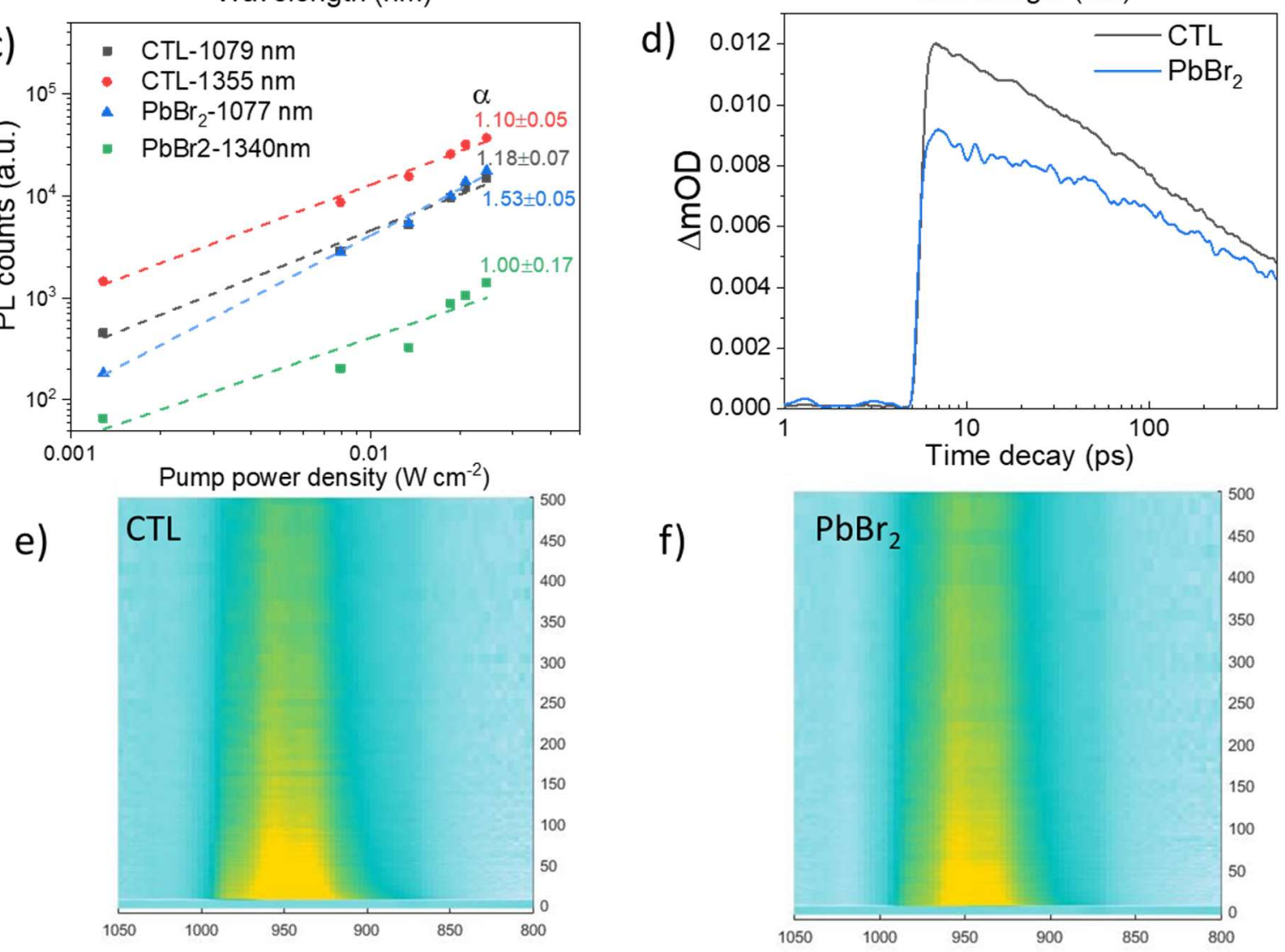

Figure 4. Decreased trap density of $\mathrm{PbBr}_{2}-\mathrm{PbS} \mathrm{CQD}$ films due to improved surface passivation. $\mathrm{PL}$ measurements of a) CTL-PbS and b) $\mathrm{PbBr}_{2}-\mathrm{PbS}$ films changing excitation (at $500 \mathrm{~nm}$ ) intensity from 0.001 to $0.024 \mathrm{~W} \mathrm{~cm}^{-2}$; c) PL intensity as a function of excitation 
intensities of the bands of CTL-PbS and $\mathrm{PbBr}_{2}-\mathrm{PbS}$ CQD films extracted from a) and $b$ ); ultrafast TA measurements: d) band-edge bleach dynamics extracted from transient absorption of e) CTL-PbS and f) $\mathrm{PbBr}_{2}-\mathrm{PbS}$ films pulsed at $1250 \mathrm{~nm}(0.99 \mathrm{eV})$ to excite the ground-totrap-state.

To study the main source of trap states in halide-passivated CQD solids, we carried out PL measurements on the films changing the excitation intensity from 0.001 to $0.024 \mathrm{~W} \mathrm{~cm}^{-2}$. CTL-PbS films show two bands of emission: the short-wavelength emission is attributed to the band edge emission, while the longer wavelength peak is from the trap emission (Figure 4a). On the contrary, $\mathrm{PbBr}_{2}-\mathrm{PbS}$ films show a sharp band edge emission at $1077 \mathrm{~nm}$ (Figure $4 \mathbf{b}$ ), with very weak broad trap emission at $1340 \mathrm{~nm}$. This indicates a drastic reduction in the number of trap states through $\mathrm{PbBr}_{2}$ reconstruction, in agreement with the FET results above. We also analyzed the recombination mechanisms using a general power law

$$
I_{P L} \propto P^{\alpha}
$$

where $I_{P L}$ is the PL intensity; the exponent $\alpha$, depending on the radiative recombination mechanisms, is expected to be close to 1 for exciton recombination and 2 for free carrier recombination. ${ }^{44}$

We plotted the PL intensities of band edge emission and trap emission for both CTL$\mathrm{PbS}$ and $\mathrm{PbBr}_{2}-\mathrm{PbS} \mathrm{CQD}$ films as a function of excitation intensity and extracted the $\alpha$ value from the linear fitting. For CTL-PbS and $\mathrm{PbBr}_{2}-\mathrm{PbS}$ films, both band edge emission $(\sim 1080 \mathrm{~nm})$ and trap emission $(\sim 1350 \mathrm{~nm})$ intensities increased with increasing the excitation intensity with a similar $\alpha$ value (Figure $4 \mathrm{c})$. When the averaged dimer-dimer distance $(\sim 15 \mathrm{~nm}$, calculated from the concentration of dimers, $\sim 1$ per 100 QDs) is much smaller than the carrier diffusion length $(\sim 120 \mathrm{~nm})$ in CTL-QD solid, the dimer would have quenched most of the PL emissions from band edge efficiently, indicating that the trap emission is mainly attributed to surface defects rather than dimers.

We also performed ultrafast TA measurements to track the charge-carrier detrapping kinetics. We pumped the CQD films using a sub-band-gap laser pulse at $1250 \mathrm{~nm}(0.99 \mathrm{eV})$ to excite the ground-to-trap-state transition and then tracked the dynamic occupation of the trap 
and band-edge states from $800 \mathrm{~nm}$ to $1050 \mathrm{~nm}$ (Figure 4d, 4e, 4f). We observed that the bandedge bleach feature subsequently grows within 10 ps (Figure 4d) as the intensity of the trapstate bleach feature decreases for both CTL-PbS and $\mathrm{PbBr}_{2}-\mathrm{PbS}$ film. When the trap state is selectively excited, the bleach signal of band-edge is immediately observed, indicating that the trap excitation involves an electron or hole in a core band-edge level of a typical QD, ${ }^{20}$ indicating that the trap states in the CTL-PbS films are mainly from QD surface defects, in agreement with the PL results.

a)
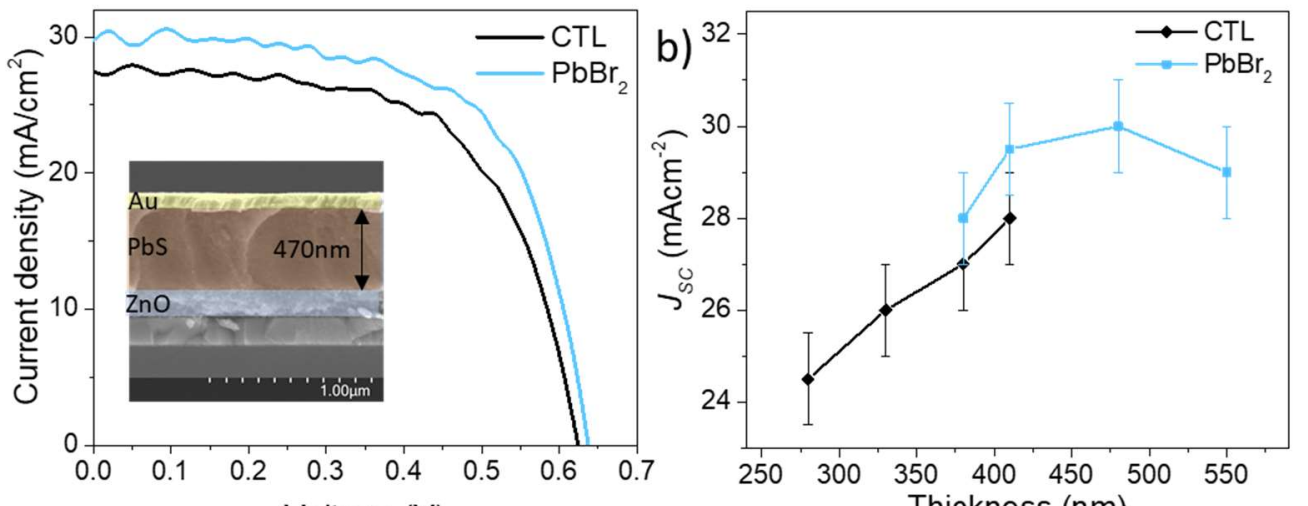

c)
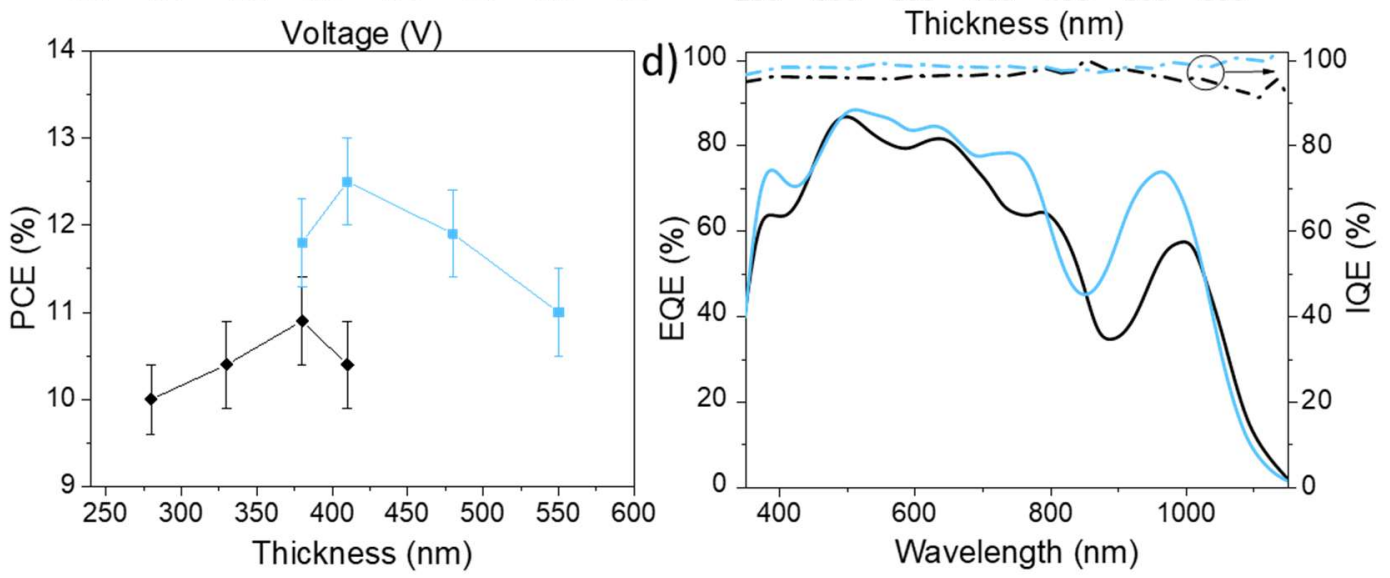

Figure 5. PV device architecture and performance. a) Device architecture and crosssectional SEM image of the best $\mathrm{PbBr}_{2}-\mathrm{PbS}$ CQD film solar cell and the $\mathrm{J}-\mathrm{V}$ characteristics under AM1.5G; thickness-dependent $J_{S C}$ (b) and PCE (c) of CTL and $\mathrm{PbBr}_{2} \mathrm{PbS}$ CQD films. d)EQE curves and IQE curves of optimal PbS CQD solar cell devices; 
We then fabricated solar cells using CTL-PbS and $\mathrm{PbBr}_{2}-\mathrm{PbS}$ CQDs (Figure 5a inset). We first investigated the thickness-dependent current density $\left(J_{S C}\right)$ and power conversion efficiency (PCE) for the CTL and $\mathrm{PbBr}_{2}-\mathrm{PbS}$ CQDs (Figures 5b, 5c). The longer $L_{d}$ of the $\mathrm{PbBr}_{2}-\mathrm{PbS} \mathrm{CQDs}$ enables the fabrication of significantly thicker active layers when compared to the CTL-PbS CQDs. We obtained the best-performing $\mathrm{PbBr}_{2}-\mathrm{PbS}$ devices when the active layer thickness reaches $420 \mathrm{~nm}$ (Figure 5a shows $470 \mathrm{~nm}$ of $\mathrm{PbS}$ film including $\sim 50 \mathrm{~nm}$ of EDT exchanged PbS CQD film as a hole transport layer); in contrast, CTL devices show an optimized $J_{S C}\left(\sim 27.5 \mathrm{~mA} \mathrm{~cm}^{-2}\right)$ and PCE $(\sim 10.9 \%)$ at the optimal thickness of $\sim 370 \mathrm{~nm}$, in agreement with previous reports. ${ }^{7}$ We note that $\mathrm{PbBr}_{2}$ reconstruction increases $J_{S C}$ and optimal thickness without losing $F F$ and $V_{O C}$. The optimal $\mathrm{PbBr}_{2}-\mathrm{PbS}$ film produces a higher $J_{S C}$ and $V_{O C}$ and thus reaching a PCE of $12.5 \%$.

The $V_{O C}, J_{S C}$, and $F F$ of the $\mathrm{PbBr}_{2}-\mathrm{PbS}$ devices are $0.64 \mathrm{~V}, 29.5 \pm 1 \mathrm{~mA} \mathrm{~cm}{ }^{-2}$, and $66 \pm 1 \%$, respectively (Figure 5a). The best CTL-PbS device has a PCE as high as $10.9 \%$, with a $V_{O C}, J_{S C}$, and $F F$ at $0.62 \mathrm{~V}, 27.5 \pm 1 \mathrm{~mA} \mathrm{~cm}^{-2}$, and $64 \pm 1 \%$, respectively. The increased $J_{S C}$ for $\mathrm{PbBr}_{2}-\mathrm{PbS}$ film is consistent with the enhanced external quantum efficiency (EQE) in Figure 5d, with excitonic peak values of $\sim 80 \%$ and integrated $J_{S C}$ value of $29.5 \mathrm{~mA} \mathrm{~cm}{ }^{-2}$ (Figure S9). We also calculated the internal quantum efficiency (IQE) using the measured EQE at $0 \mathrm{~V}$ bias and $-2 \mathrm{~V}$ bias in the CQD active layer (Figure 5d), where $\mathrm{PbBr}_{2}-\mathrm{PbS}$ film showed enhanced IQE compared to CTL-PbS films, especially in the excitonic tail region. This enhancement agrees with the interpretation that fewer trap states and longer carrier lifetime contributes to longer carrier diffusion lengths and thus enables efficient extraction from a thicker active layer.

In conclusion, we report a CQD reconstruction to improve the $\mathrm{PbS} C Q \mathrm{D}$ quality to achieve high PLQY, peak-to-valley ratio, narrow HWHM, decreased dimers, and reduced surface traps, resulting in photovoltaic performance with improvements in all photovoltaic parameters (Voc, JSc, and $F F$ ). We mapped the density of trap states, observing a lower density of trap states, attributed to the improved surface passivation, demonstrated by the intensity-dependent PL measurements and ultrafast TA results. The resultant longer carrier lifetime is achieved by improving surface passivation and thus improved diffusion length. The CQD reconstruction improves the CQD quality, a further path to high performance CQD solar cells and lightemitting diodes. 
AUTHOR INFORMATION

Corresponding Author

*E-mail: ted.sargent@utoronto.ca.

\section{ORCID}

Bin Sun: 0000-0002-8233-0999

Mingyang We: 0000-0003-4820-2210

Yitong Dong: 0000-0002-7069-3725

Laxmi Kishore Sagar: 0000-0002-7656-7308

Oleksandr Voznyy: 0000-0002-8656-5074

Frédéric Laquai: 0000-0002-5887-6158

Zhenghong Lu: 0000-0003-2050-0822

F. Pelayo García de Arquer: 0000-0003-2422-6234

Edward H. Sargent: 0000-0003-0396-6495

\section{Acknowledgements}

This work was supported by Ontario Research Fund-Research Excellence program (ORF7Ministry of Research and Innovation, Ontario Research Fund-Research Excellence Round 7), by the King Abdullah University of Science and Technology (KAUST) Office of Sponsored Research (OSR) under Award No. OSR-2018-CRG7-373702 and Award No. OSR-2018-CARF/CCF-3079, and by the Natural Sciences and Engineering Research Council (NSERC) of Canada. The authors acknowledge the financial support from QD Solar.

References

(1) Murray, C. B.; Norris, D. J.; Bawendi, M. G., Synthesis and characterization of nearly monodisperse $\mathrm{CdE}$ ( $\mathrm{E}=$ sulfur, selenium, tellurium) semiconductor nanocrystallites. J. Am. Chem. Soc. 1993, 115 (19), 8706-8715.

(2) Yu, W. W.; Wang, Y. A.; Peng, X., Formation and Stability of Size-, Shape-, and Structure-Controlled CdTe Nanocrystals: Ligand Effects on Monomers and Nanocrystals. Chem. Mater. 2003, 15 (22), $4300-$ 4308. 
(3) Murray, C. B.; Kagan, C. R.; Bawendi, M. G., Synthesis and characterization of monodisperse nanocrystals and close-packed nanocrystal assemblies. Annual Review of Materials Science 2000, 30 , 545-610.

(4) Sargent, E. H., Colloidal quantum dot solar cells. Nat Photon 2012, 6 (3), 133-135.

(5) Park, Y. I.; Piao, Y.; Lee, N.; Yoo, B.; Kim, B. H.; Choi, S. H.; Hyeon, T., Transformation of hydrophobic iron oxide nanoparticles to hydrophilic and biocompatible maghemite nanocrystals for use as highly efficient MRI contrast agent. J. Mater. Chem. 2011, 21 (31), 11472-11477.

(6) Kagan, C. R.; Lifshitz, E.; Sargent, E. H.; Talapin, D. V., Building devices from colloidal quantum dots. Science 2016, 353 (6302).

(7) Liu, M.; Voznyy, O.; Sabatini, R.; Garcia de Arquer, F. P.; Munir, R.; Balawi, A. H.; Lan, X.; Fan, F.; Walters, G.; Kirmani, A. R.; Hoogland, S.; Laquai, F.; Amassian, A.; Sargent, E. H., Hybrid organicinorganic inks flatten the energy landscape in colloidal quantum dot solids. Nat Mater 2017, 16 (2), 258263.

(8) Sun, B.; Voznyy, O.; Tan, H.; Stadler, P.; Liu, M.; Walters, G.; Proppe, A. H.; Liu, M.; Fan, J.; Zhuang, T.; Li, J.; Wei, M.; Xu, J.; Kim, Y.; Hoogland, S.; Sargent, E. H., Pseudohalide-Exchanged Quantum Dot Solids Achieve Record Quantum Efficiency in Infrared Photovoltaics. Adv Mater 2017, 29 (27), 1700749.

(9) Xu, J.; Voznyy, O.; Liu, M.; Kirmani, A. R.; Walters, G.; Munir, R.; Abdelsamie, M.; Proppe, A. H.; Sarkar, A.; Garcia de Arquer, F. P.; Wei, M.; Sun, B.; Liu, M.; Ouellette, O.; Quintero-Bermudez, R.; Li, J.; Fan, J.; Quan, L.; Todorovic, P.; Tan, H.; Hoogland, S.; Kelley, S. O.; Stefik, M.; Amassian, A.; Sargent, E. H., 2D matrix engineering for homogeneous quantum dot coupling in photovoltaic solids. Nat Nanotechnol 2018, 13 (6), 456-462.

(10) Kim, J.; Ouellette, O.; Voznyy, O.; Wei, M.; Choi, J.; Choi, M.-J.; Jo, J. W.; Baek, S.-W.; Fan, J.; Saidaminov, M. I.; Sun, B.; Li, P.; Nam, D.-H.; Hoogland, S.; Lu, Z.-H.; García de Arquer, F. P.; Sargent, E. H., Butylamine-Catalyzed Synthesis of Nanocrystal Inks Enables Efficient Infrared CQD Solar Cells. 2018, 30 (45), 1803830.

(11) Chuang, C.-H. M.; Brown, P. R.; Bulović, V.; Bawendi, M. G., Improved performance and stability in quantum dot solar cells through band alignment engineering. Nature Materials 2014, 13, 796.

(12) Choi, M. J.; Garcia de Arquer, F. P.; Proppe, A. H.; Seifitokaldani, A.; Choi, J.; Kim, J.; Baek, S. W.; Liu, M.; Sun, B.; Biondi, M.; Scheffel, B.; Walters, G.; Nam, D. H.; Jo, J. W.; Ouellette, O.; Voznyy, O.; Hoogland, S.; Kelley, S. O.; Jung, Y. S.; Sargent, E. H., Cascade surface modification of colloidal quantum dot inks enables efficient bulk homojunction photovoltaics. Nat Commun 2020, 11 (1), 103.

(13) Chuang, C. H.; Maurano, A.; Brandt, R. E.; Hwang, G. W.; Jean, J.; Buonassisi, T.; Bulovic, V.; Bawendi, M. G., Open-circuit voltage deficit, radiative sub-bandgap states, and prospects in quantum dot solar cells. Nano Lett 2015, 15 (5), 3286-94.

(14) Zhitomirsky, D.; Kramer, I. J.; Labelle, A. J.; Fischer, A.; Debnath, R.; Pan, J.; Bakr, O. M.; Sargent, E. H., Colloidal quantum dot photovoltaics: the effect of polydispersity. Nano Lett 2012, 12 (2), 1007-12.

(15) Zhitomirsky, D.; Voznyy, O.; Levina, L.; Hoogland, S.; Kemp, K. W.; Ip, A. H.; Thon, S. M.; Sargent, E. H., Engineering colloidal quantum dot solids within and beyond the mobility-invariant regime. Nature Communications 2014, 5, 3803.

(16) Voznyy, O.; Levina, L.; Fan, J. Z.; Askerka, M.; Jain, A.; Choi, M.-J.; Ouellette, O.; Todorović, P.; Sagar, L. K.; Sargent, E. H., Machine Learning Accelerates Discovery of Optimal Colloidal Quantum Dot Synthesis. ACS Nano 2019, 13 (10), 11122-11128.

(17) Ip, A. H.; Thon, S. M.; Hoogland, S.; Voznyy, O.; Zhitomirsky, D.; Debnath, R.; Levina, L.; Rollny, L. R.; Carey, G. H.; Fischer, A.; Kemp, K. W.; Kramer, I. J.; Ning, Z.; Labelle, A. J.; Chou, K. W.; Amassian, A.; Sargent, E. H., Hybrid passivated colloidal quantum dot solids. Nat Nano 2012, 7 (9), 577 582. 
(18) Tang, J.; Kemp, K. W.; Hoogland, S.; Jeong, K. S.; Liu, H.; Levina, L.; Furukawa, M.; Wang, X.; Debnath, R.; Cha, D.; Chou, K. W.; Fischer, A.; Amassian, A.; Asbury, J. B.; Sargent, E. H., Colloidalquantum-dot photovoltaics using atomic-ligand passivation. Nat Mater 2011, 10 (10), 765-771.

(19) Winslow, S. W.; Liu, Y.; Swan, J. W.; Tisdale, W. A., Quantification of a PbClx Shell on the Surface of PbS Nanocrystals. ACS Materials Letters 2019, 1 (2), 209-216.

(20) Gilmore, R. H.; Liu, Y.; Shcherbakov-Wu, W.; Dahod, N. S.; Lee, E. M. Y.; Weidman, M. C.; Li, H.; Jean, J.; Bulović, V.; Willard, A. P.; Grossman, J. C.; Tisdale, W. A., Epitaxial Dimers and Auger-Assisted Detrapping in PbS Quantum Dot Solids. Matter 2019, 1 (1), 250-265.

(21) Zherebetskyy, D.; Scheele, M.; Zhang, Y.; Bronstein, N.; Thompson, C.; Britt, D.; Salmeron, M.; Alivisatos, P.; Wang, L. W., Hydroxylation of the surface of PbS nanocrystals passivated with oleic acid. Science 2014, 344 (6190), 1380-4.

(22) Yuan, M.; Liu, M.; Sargent, E. H., Colloidal quantum dot solids for solution-processed solar cells. Nat Energy 2016, 1, 16016.

(23) Lan, X.; Voznyy, O.; García de Arquer, F. P.; Liu, M.; Xu, J.; Proppe, A. H.; Walters, G.; Fan, F.; Tan, H.; Liu, M.; Yang, Z.; Hoogland, S.; Sargent, E. H., 10.6\% Certified Colloidal Quantum Dot Solar Cells via Solvent-Polarity-Engineered Halide Passivation. Nano Letters 2016, 16 (7), 4630-4634.

(24) Cao, Y. M.; Stavrinadis, A.; Lasanta, T.; So, D.; Konstantatos, G., The role of surface passivation for efficient and photostable PbS quantum dot solar cells. Nat Energy 2016, 1, 16035.

(25) Wang, Y.; Lu, K.; Han, L.; Liu, Z.; Shi, G.; Fang, H.; Chen, S.; Wu, T.; Yang, F.; Gu, M.; Zhou, S.; Ling, X.; Tang, X.; Zheng, J.; Loi, M. A.; Ma, W., In Situ Passivation for Efficient PbS Quantum Dot Solar Cells by Precursor Engineering. Adv Mater 2018, 30 (16), e1704871.

(26) Zhang, J.; Gao, J.; Miller, E. M.; Luther, J. M.; Beard, M. C., Diffusion-controlled synthesis of PbS and PbSe quantum dots with in situ halide passivation for quantum dot solar cells. ACS Nano 2014, 8 (1), 614-22.

(27) Moreels, I.; Lambert, K.; Smeets, D.; De Muynck, D.; Nollet, T.; Martins, J. C.; Vanhaecke, F.; Vantomme, A.; Delerue, C.; Allan, G.; Hens, Z., Size-Dependent Optical Properties of Colloidal PbS Quantum Dots. ACS Nano 2009, 3 (10), 3023-3030.

(28) Wang, Y.; Liu, Z.; Huo, N.; Li, F.; Gu, M.; Ling, X.; Zhang, Y.; Lu, K.; Han, L.; Fang, H.; Shulga, A. G.; Xue, Y.; Zhou, S.; Yang, F.; Tang, X.; Zheng, J.; Antonietta Loi, M.; Konstantatos, G.; Ma, W., Roomtemperature direct synthesis of semi-conductive PbS nanocrystal inks for optoelectronic applications. Nature Communications 2019, 10 (1), 5136.

(29) Shimpi, J. R.; Sidhaye, D. S.; Prasad, B. L. V., Digestive Ripening: A Fine Chemical Machining Process on the Nanoscale. Langmuir 2017, 33 (38), 9491-9507.

(30) Zhang, C.; Xia, Y.; Zhang, Z.; Huang, Z.; Lian, L.; Miao, X.; Zhang, D.; Beard, M. C.; Zhang, J., Combination of Cation Exchange and Quantized Ostwald Ripening for Controlling Size Distribution of Lead Chalcogenide Quantum Dots. Chemistry of Materials 2017, 29 (8), 3615-3622.

(31) Razgoniaeva, N.; Yang, M.; Garrett, P.; Kholmicheva, N.; Moroz, P.; Eckard, H.; Royo Romero, L.; Porotnikov, D.; Khon, D.; Zamkov, M., Just Add Ligands: Self-Sustained Size Focusing of Colloidal Semiconductor Nanocrystals. Chemistry of Materials 2018, 30 (4), 1391-1398.

(32) Dagtepe, P.; Chikan, V., Quantized Ostwald Ripening of Colloidal Nanoparticles. The Journal of Physical Chemistry C 2010, 114 (39), 16263-16269.

(33) Hines, M. A.; Scholes, G. D., Colloidal PbS nanocrystals with size-tunable near-infrared emission: Observation of post-synthesis self-narrowing of the particle size distribution. Advanced Materials 2003, 15 (21), 1844-1849.

(34) Kirkwood, N.; Monchen, J. O. V.; Crisp, R. W.; Grimaldi, G.; Bergstein, H. A. C.; du Fossé, I.; van der Stam, W.; Infante, I.; Houtepen, A. J., Finding and Fixing Traps in II-VI and III-V Colloidal Quantum Dots: The Importance of Z-Type Ligand Passivation. Journal of the American Chemical Society 2018, 140 (46), 15712-15723. 
(35) Seth, J.; Prasad, B. L. V. J. N. R., Bromide ion mediated modification to digestive ripening process: Preparation of ultra-small Pd, Pt, Rh and Ru nanoparticles. 2016, 9 (7), 2007-2017.

(36) Weidman, M. C.; Beck, M. E.; Hoffman, R. S.; Prins, F.; Tisdale, W. A., Monodisperse, Air-Stable PbS Nanocrystals via Precursor Stoichiometry Control. ACS Nano 2014, 8 (6), 6363-6371.

(37) Klimov, V. I.; Mikhailovsky, A. A.; McBranch, D. W.; Leatherdale, C. A.; Bawendi, M. G., Mechanisms for intraband energy relaxation in semiconductor quantum dots: The role of electron-hole interactions. Phys. Rev. B 2000, 61 (20), R13349-R13352.

(38) Klimov, V. V.; Mikhailovsky, A. A.; McBranch, D. W.; Leatherdale, C. A.; Bawendi, M. G., Quantization of multiparticle auger rates in semiconductor quantum dots. Science 2000, 287 (5455), 1011-3.

(39) Carey, G. H.; Levina, L.; Comin, R.; Voznyy, O.; Sargent, E. H., Record Charge Carrier Diffusion Length in Colloidal Quantum Dot Solids via Mutual Dot-To-Dot Surface Passivation. Adv Mater 2015, 27 (21), 3325-30.

(40) Proppe, A. H.; Xu, J.; Sabatini, R. P.; Fan, J. Z.; Sun, B.; Hoogland, S.; Kelley, S. O.; Voznyy, O.; Sargent, E. H., Picosecond Charge Transfer and Long Carrier Diffusion Lengths in Colloidal Quantum Dot Solids. Nano Letters 2018, 18 (11), 7052-7059.

(41) Dong, Q.; Fang, Y.; Shao, Y.; Mulligan, P.; Qiu, J.; Cao, L.; Huang, J., Electron-hole diffusion lengths \&gt; $175 \mu \mathrm{m}$ in solution-grown $\mathrm{CH}<$ sub $>3</$ sub $>\mathrm{NH}<$ sub $>3</$ sub $>$ Pbl $<$ sub $>3</$ sub $>$ single crystals. 2015, 347 (6225), 967-970.

(42) Stadler, P.; Sutherland, B. R.; Ren, Y.; Ning, Z.; Simchi, A.; Thon, S. M.; Hoogland, S.; Sargent, E. H., Joint Mapping of Mobility and Trap Density in Colloidal Quantum Dot Solids. ACS Nano 2013, 7 (7), 5757-5762.

(43) Oh, S. J.; Straus, D. B.; Zhao, T.; Choi, J. H.; Lee, S. W.; Gaulding, E. A.; Murray, C. B.; Kagan, C. R., Engineering the surface chemistry of lead chalcogenide nanocrystal solids to enhance carrier mobility and lifetime in optoelectronic devices. Chem. Commun. 2017, 53 (4), 728-731.

(44) Schmidt, T.; Lischka, K.; Zulehner, W., Excitation-power dependence of the near-band-edge photoluminescence of semiconductors. Physical Review B 1992, 45 (16), 8989-8994. 